\title{
LA PRESENCIA MASCULINA EN LOS CUENTOS DE RIMA DE VALLBONA
}

\author{
Mary Gómez-Parham \\ University of Houston, Downtown \\ Ingrid Hansz \\ Rice University
}

El título de este trabajo menciona un tema-cómo aparecen los hombres en la obra literaria de las mujeres - el cual ha sido tratado con sorprendente negligencia. Los investigadores tradicionales han pasado décadas reflexionando sobre los matices de los retratos hechos por los hombres de sus Emas y de sus Anas. Más recientemente las investigadoras feministas han publicado ensayos acerca de la naturaleza anti-feminista sobre dichos retratos, o se han volcado a "trabajos por mujeres con el fin de encontrar representaciones alternas de las mujeres. .." (Heilbrun y Higonet xv). Estos estudios de cómo escriben las mujeres, cómo perciben el mundo o cómo ven sus vidas, han jugado un papel invalorable en el estudio de la obra literaria femenina. ${ }^{1}$ Pero el retrato de los hombres hechos por las mujeres, cuya contraparte ha sido considerada por tanto tiempo como un área legítima de investigación, no ha sido todavía estudiado con mucha profundidad. Los libros que tratan este tema se limitan principalmente a Der Typus des Mannes in der Dichtung der Frau por Else Hoppe y el más reciente de Jane Miller, Women Writing About Men. La crítica Elizabeth Ordóñez se lamenta de esta situación en forma indirecta, rechazando la noción de que solamente (o aun principalmente) los personajes femeninos deberían ser de interés de los investigadores de la obra femenina, al preguntar retóricamente, "(W)hy should a woman writer's treatment of the male be less relevant for feminist theory than her writing of the female?" ( “Por qué debería ser menos importante para la teoría feminista el tratamiento del hombre por una escritora que su obra literaria sobre la mujer?”) (118). Ella cree que “(c)onsiderations of questions such as this might result in the uncovering of unspoken notions about gender formation and might comment on the formulation of discourses shaping gender in texts by both men and women" ("las consideraciones de preguntas semejantes podrían tener por resultado el descubrimiento de nociones aún no expresadas sobre información del género y podría ofrecer un comentario sobre la formulación de discursos que esculpen el género en texto tanto de los hombres como de las mujeres") (118). Es de acuerdo con opiniones tales como la que expresa Ordóñez que se ha escrito este estudio sobre la presencia masculina en los cuentos cortos de Rima de Vallbona. Se espera que este trabajo sea una contribución útil al estudio de esta importante área tan dejada de lado, al mismo tiempo que proyecte una luz sobre el aspecto central del trabajo de Vallbona en particular, y por extrapolación y analogía, sobre las vidas y obra de otras mujeres, sean escritoras o no. 
La razón principal de la importancia del estudio de este tema, en general, y más específicamente en el contexto del trabajo de Vallbona, es una razón socio-crítica. Obviamente, para empezar, los hombres son importantes para las mujeres, y poder comprender las actitudes de las mujeres hacia ellos es especialmente crucial en este momento en que las mujeres quieren volver a definir quienes son vis-`a-vis quienes siempre se han encargado de definirlas. Estas actitudes contemporáneas-y, es claro, las de los hombres hacia las mujeres-son complejas y a menudo confusas. Como dice Jane Miller, hablando de las novelas de las mujeres, "(M)en are to be found in women's novels as they are to be found in women's heads and histories: equivocably. They are ourselves, our protectors, our representatives and our opponents" ("Los hombres se encuentran en las novelas de las mujeres de la misma manera que se encuentran en la cabeza de las mujeres equívocamente. Ellos son nosotras mismas, nuestros protectores, nuestros representatnes y nuestros contrincantes") (3). El resultado de esta realidad, concluye Miller, es que, "we can expect double vision and shifting ground in the novels of women" (17). ${ }^{2}$ Otro resultado, según ella, es que hoy "most women's novels are engaged at some level in extricating their authors as well as their heroines from charges of abnormality" ("la mayoría de las novelas de escritoras tratan, en algún nivel, de liberarse de sus autoras así como sus heroínas de acusaciones de anormalidad") (3). Así es que las escritoras pueden vindicar a miembros de su propio sexo al esculpir cuidadosamente a los personajes masculinos, cuyas verrugas quedan penosamente en evidencia. Estas dos características en la obra de muchas escritoras de hoy en día - una tendencia a la "doble visión" y a envolverse en una campaña de vindicación de las mujeres-son parte de las muchas relaciones de la mujer moderna con los hombres; por eso la naturaleza mimética de dicha obra le confiere una relevancia de vida real la cual sería bueno que investigáramos muy de cerca.

Las razones antes mencionadas de la necesidad de una buena revisión de los hombres creados por las escritoras, se aplica al caso de Rima de Vallbona en cuya obra se encuentran las características comunes que están presentes en obras de otras escritoras. En su trabajo encontramos evidencia de un intenso interés en los hombres, el cual es básicamente parte de un "plea for mutual understanding" ("una súplica para un entendimiento mutuo") entre los sexos, una súplica que es el motivo central de su obra (Dowling, "Point of View. . ." (71). ${ }^{3}$ El deseo ya mencionado de vindicar también se encuentra claramente presente, pero es posible que lo más importante sea la marca en Vallbona de la "doble visión" de Jane Miller: la manifestación en la obra de Vallbona de la ambivalencia de las mujeres modernas hacia los hombres en su vida.

En este ensayo, se hablará sobre los hombres de Vallbona desde el punto de vista de las características ya descritas y que son la clave para comprender su obra. Con este fin, se analizará algunos cuentos cortos representativos en lugar de entrar en sus dos novelas, ya que este enfoque permitirá un encuentro con muestras más variadas de los hombres que habitan el subconsciente de los narradores de Vallbona. Dentro de dichos cuentos, se enfocará a los personajes masculinos en papeles que parezcan más importantes para la autora-los curas, esposos, padres, hijos, hermanos y amantes. ${ }^{4}$

La figura del cura es central en varios cuentos de Vallbona, tales como "Siervo de siervos" en Polvo del camino, "Desde aquî" en Mujeres y agonías y "Cosecha de pecadores" en Cosecha de pecadores. Pero el cuento en el cual la presentación del hombre como cura es más compleja es "Lo inconfesable," en Mujeres y agonías. Es un cuento que ha evocado mucho inte- 
rés de la crítica, en gran parte como resultado de los temas que trata, es decir, la masturbación femenina y el cura lascivo e hipócrita. Por lo menos en la primera área, el cuento fue algo muy innovador, y no sólo en la literatura hispánica. Y en la segunda, se conocen muy pocos escritores, ya sean o no hispanos, que hayan manejado con sensibilidad un tema tan delicado. En "Lo inconfesable" el padre Segura narra su cuento; él es uno de varios narradores masculinos de Vallbona que llevan al lector a la mente del hombre, mientras él observa el mundo, mientras observa a las mujeres. El nos transporta a los profundidades recónditas de la mente, cuyas vueltas bizantinas son a la vez repelentes y comprensibles. El hombre es un hipócrita al tratar de convencerse a sí mismo que sus grabaciones magnetofónicas en el confesionario de las narraciones de las niñas acerca de su masturbación constituyen una investigación científica, y que su trabajo ha sido inspirado por un celo pastoral. El explota a las niñas sexualmente, engañándolas a proporcionarle a él material que sacie su apetituo sexual. Y es un misógino predecible que se refiere a "las mujeres, esas timoratas que a la hora de la tentación, no se lo piensan dos veces, pero se acercan al confesionario con remilgos, con reticencias" (89). Sin embargo es claro que Vallbona desea dar espacio para que haya exculpación, dejando que el padre Segura narre el sufrimiento de su pobreza cuando niño, su pena por su madre desgraciada, su soledad y la falta de sensibilidad e hipocresía de sus superiores del clero. Por encima de todo, Vallbona parece querer destacar la lucha que encara un hombre ordinario para lograr su voto de castidad, al hacer que él se lamente: “iSi los sacerdotes fueran un género neutro, un 'ello' sin necesidades físicas, sin deseos, sin sentir que su fisiología masculina se estremece de ansias de mujer!” (95) Esta expresión de exoneración de la dificultad en la vida del cura, contrapuesta a la revelación de sus atroces perversiones, es evidencia de los sentimientos ambivalentes hacia el hombre a los cuales se refiere Jane Miller.

Si en "Lo inconfesable" Vallbona exhibe la ambivalencia hacia los hombres de una escritora moderna, también aquí se aboca a la otra actividad común que describe Jane Miller: en realidad está vindicando a las mujeres en este retrato de un hombre confundido, neurótico. Las niñas que confiesan su "pecado" sexual al padre Segura son descritas por el superior que reprende al padre, como "Hijas de María, todas," a quienes se les requiere, en todo momento, que sean "el ejemplo máximo de pureza y castidad" (97). Pero el hecho es que las niñas no son, de la manera en que el monseñor comprende esas palabras, ni castas ni puras. Es decir que no son seres asexuales, sino seres muy sexuales que han sido llevados a ocultar su sexualidad. Una de las niñas le dice a su malicioso confesor, por ejemplo, "(D)icen que sólo los hombres lo practican (la masturbación): yo me sentía doblemente culpable, creía que era yo sola. . ." (98). La niña se siente doblemente culpable porque ha cometido no sólo el pecado en cuestión sino también ha tenido demasiado vergüenza para confesarlo. El mensaje que se encuentra aquí es que la represión tradicional de las mujeres y/o el ocultar su sexualidad por temor a la reprensión, violación, embarazo o al beso de la muerte de la sociedad (y aun físico) por la pérdida de la virginidad, es comprensible y lamentable. Acá tenemos a un grupo de jovencitas que se atreven a ser honestas sobre su sexualidad, bajo las condiciones más sancionables socialmente, y el oído al otro lado de la ventanilla del confesionario resulta ser de un hombre que las hace víctimas sexualmente.

Esta ambivalencia de parte de la autora en su trato del padre Segura, sin embargo, está completamente ausente en el caso del esposo y del hermano en "Infame retorno" en Cosecha 
de pecadores. El esposo de la protagonista es un canalla y objeto de su sorna y rabia, como son casi todos los esposos en los cuentos de Vallbona. En este cuento en particular, el ex-esposo deja de mantener económicamente a su hijo y es una figura fría y cruel que, por casualidad, ha traído a su esposa la noticia de la muerte de su amiga Gladys:

\begin{abstract}
¡Ah! lo imperdonable es que sea siempre la voz cruda de Oscar ... la que traiga las muertes de los seres queridos por los hilos telefónicos. Hace cinco años también me dijo: "Ha muerto Hernán ... Sí, lo que te digo, que ha muerto tu hermano", así como así, igual que si hubiese dicho que se había descompuesto la nevera ... (57)
\end{abstract}

La crueldad del esposo y el odio que siente su mujer a esa crueldad son evidentes en el pasaje anterior. En cuanto al hermano muerto a quien la autora coloca junto a la amiga Gladys entre los "seres queridos", resulta ser en realidad un individuo explotador que agota a la narradora de manera emocional y económica. Más importante aún, él ha tratado de socavar la fé religiosa que él sabe que la narradora desea conservar desesperadamente. Es cruel e implacable en su empeño, y su constante explotación y falta de sensibilidad hacen que la narradora termine su relación con él, declarando finalmente, "'Has muerto para mí desde ahora, Hernán, y desde ahora te entierro para siempre"" (58). Al final, en un giro Borgesiano, este "ser querido" precipita la ruina de su hermana con la destrucción de su tesis doctoral.

En "Infame retorno" hay algo insólito, sin embargo, en el trato de Vallbona de sus personajes masculinos, ya que, como se ha indicado antes, ellos son retratados en esta historia sin la ambivalencia que se nota en "Lo inconfesable" y en otros cuentos, como en "Más allá de la carne" de Cosecha de pecadores. En este último, así como en "Infame retorno," un hombre es finalmente la fuente de la destrucción de la mujer, al transformarse su amante en su asesino. El mensaje es: los hombres pueden ser peligrosos e indignos de confianza. Sin embargo, y esto es muy importante en una visión general de la obra literaria de Vallbona, los hombres son representados al mismo tiempo como irresistiblemente atractivos. Son necesarios para la satisfacción sexual, estos hombres, en palabras de la narradora, "olorosos a sudor y a hombre-hombre . . . (que) colman mi cuerpo de ellos hasta el éxtasis supremo" (30). Estos son los hombres-comoamantes que aparecen a menudo en los cuentos de Vallbona, trayendo gratificación a las mujeres por medio de su sexualidad o, de vez en cuando, a través de sólo el cariño. Ellos ofrecen sexo (que aquí la autora llama "amor" como lo hace a menudo en su obra) el cual es descrito por la protagonista de "Más allá" como "placer intenso y no costumbre, repetida costumbre de contrato matrimonial" (30). Este pasaje otra vez se dirige a los esposos insensibles como Oscar en "Infame retorno" mientras que, simultáneamente, vindica a aquellas mujeres cuyos maridos ineptos en la sexualidad las fuerzan a buscar gratificación sexual en el adulterio. Así, "Más allá" exhibe tanto la ambivalencia como el deseo-de-vindicar que se ha encontrado como característica del trato de Vallbona de su hombre de ficción.

Otro cuento que tiene que ver con la figura del amante es "La parábola del edén imposible" en Mujeres y agonías. Aquí el hombre se convierte en algo más que meramente necesario para la felicidad sexual; aparenta convertirse en el posible-salvador de la mujer en otras áreas, una fuente imaginada de satisfacción y consumación en un sentido menos carnal. La protagonista de "La parábola" parece esperar que el héroe la salve no sólo del tedio sexual sino de la 
insensatez y aburrimiento de un matrimonio desgraciado y una existencia generalmente trivial. ${ }^{5}$ Y él hace esto, por lo menos temporariamente: él es quien le trae a ella "la flor del éxtasis" (24), "el único que podría construir la nave, izar las velas rumbo al paraíso-plenitud, y llegar" (23), quien la lleva de "su ahora, su limpia-casa, barre-suelos, cambia-pañales, lavaplatos, marido-enla-cama, y final-de-día-vacío-rota-toda-por-dentro” (21). El tema de consumación que se encuentra en su obra literaria está claramente presente aquí: las mujeres que han experimentado esta gratificación proporcionada por un hombre-amante (o tal vez hasta un marido; el punto no queda claro) son "mujeres completas" (22), y la narradora, después de la consumación de su asunto amoroso se da cuenta de que "se sintió completa; por primera vez se sintió mujer" (24). El amor de un hombre y un buen sexo, entonces, puede traer estimulación y alegría, así como algún grado de satisfacción, para las mujeres. Sin embargo, la ambivalencia de Vallbona, tanto aquí como en otras obras, impide un endoso en pleno del hombre en consideración. Aunque el amante de la protagonista no le hace daño físico, como en el caso de "Más allá," el cuento termina en realidad con una nota de desilusión, al darse cuenta la heroína de que su héroe no es un salvador y ella empieza a añorar "la orilla que una tarde abandonó llena de esperanzas . .." (25). En suma, curas, hermanos y maridos tienen sin duda alguna, un efecto pernicioso en la vida de las mujeres, mientras que el amante puede ser una fuente de algún placer y satisfacción, aunque tal vez no todo lo que ellas quisieran: este es el mensaje de "Parábola," y los aspectos positivos de este personaje masculino, el amante, son aún más fuertes y evidentes en de Vallbona.

Lamentablemente no tenemos ahora suficiente tiempo para discutir estos y otros cuentos en mayor detalle. Sin embargo, los que acá se han presentado contienen evidencia de un molde diferente en el tratamiento de Rima de Vallbona del personaje masculino_el mismo molde de ambivalencia y reinvindicación que está presente en la obra literaria de muchas escritoras contemporáneas.

\section{Notas}

1 Algunos de los más importantes de estos trabajos son los de Harwick, Moers, Spacks, Ellmann, Rogers, Heilbrun and Higonnet, Cornillon, Showalter, Gilbert and Gubar, Beth Miller, y Mora and Van Hooft.

En su libro Archetypal Patterns in Women's Fiction, Annis Pratt ofrece tanto la confirmación de la teoría de Jane Miller acerca de la presencia de ambivalencia del autor en la ficción femenina como una explicación muy convincente de por qué esta ambivalencia es tan frecuente en dicha ficción. Ella escribe que,

the novel is a social construct and ... women authors internalize gender norms from birth. A battle goes on unremittingly within the heads of most women authors between assumptions about male and female behavior norms and dreams of more gender-free possibilities. It is as if the authorial superego, or censor, preoccupied with proper conduct for women, were constantly represssing subversive desires for self-expression and development. The disjunction between an author's social conscience and her need for selfhood renders characterization ambivalent, tone and attitude ambiguous, and plots problematic. (11) na: Desafíos ideológicos y perspectiva de la narración en su obra literaria."

En este trabajo, se usará el término "amante" para referirse a objetos-de-amor, haya o no una relación sexual entre las dos personas mencionadas. 
El tema del tedio emocional y sexual que puede traer el matrimonio es tratado en forma interesante en "Iniciación" en Cosecha de pecadores, en el cual un hijo se da cuenta, inesperadamente, de la frialdad de la relación entre sus padres.

\section{Bibliografía}

Cornillon, Susan Koppelman (ed.). 1972. Images of Women in Fiction: Feminist Perspectives. Bowling Green: Bowling Green University Popular Press.

Dowling, Lee. 1985. "Point of View in Rima de Vallbona's Novel Las sombras que perseguimos." Revista Chicano-Riqueña. 13(1): 64-73.

Dowling, Lee. 1986. "Rima de Vallbona: Desafíos ideológicos y perspectiva de la narración en su obra literaria." Letras. 11(12): 193-214.

Ellmann, Mary. 1968. Thinking About Women. New York: Harcourt Brace Jovanovich.

Friedan, Betty. 1963. The Feminine Mystique. New York: W. W. Norton.

Freud, Sigmund. 1954. The Origins of Psychoanalysis: Letters to Wilhelm Fliess, Drafts and Notes: 1887-1902. New York: Basic Books.

Freud, Sigmund. 1962. Three Essays on the Theory of Sexuality. New York: Avon Books.

Gilbert, Susan M. y Susan Gubart. 1979. The Madwoman in the Attic: The Woman Writer and the Nineteenth-Century Literary Imagination. New Haven: Yale UP.

Hardwick, Elizabeth. 1974. Seduction and Betrayal: Women and Literature. New York: Random House. (publicado en 1975 por Vintage Books).

Heilbrun, Carolyn G.y Margaret R. Higonnet (eds.). 1983. The Representation of Women in Fiction. Baltimore: The Johns Hopkins UP.

Hoppe, Elsa (ed.). 1960. Der Typus des Mannes in der Dichtung der Frau. Hamburg: Marion von Schröder Verlag. (Traducido al español por Inés Durruty y publicado como El hombre en la literatura de la mujer. Madrid: Gredos, 1964).

Miller, Beth (ed.). 1983. Women in Hispanic Literature: Icons and Fallen Idols. Berkeley: University of California Press.

Miller, Jane. 1986.Women Writing About Men. New York: Pantheon Books.

Moers, Ellen. 1976. Literary Women: The Great Writers. Garden City, N.Y.: Doubleday. 
Mora, Gabriela y Karen S. Van Hooft. 1982. Theory and Practice of Feminist Literary Criticism. Ypsilanti: Bilingual Press.

Ordóñez, Elizabeth J. 1989. Rev. of Contemporary Women Writers of Spain, by Janet Pérez. South Central Review. 6 (1): 116-18.

Owen, Ursula. 1983. Fathers: Reflections by Daughters. London: Virago Press (también publicado por Pantheon Books en 1985).

Pratt, Annis. 1981. Archetypal Patterns in Women's Fiction. Bloomington: Indiana University Press.

Rogers, Katharine M. 1966. The Troublesome Helpmate: A History of Misognyny in Literature. Seattle : University of Washington Press

Showalter, Elaine (ed.). 1985. The New Feminist Criticism: Essays on Women, Literature and Theory. New York: Pantheon Books.

Spacks, Patricia Meyer. 1975. The Female Imagination. New York: Alfred A. Knopf

Vallbona, Rima de. 1981. Cosecha de pecadores. San José: Editorial Costa Rica.

Vallbona, Rima de. 1982. Mujeres y agonías. Houston: Arte Público Press

Vallbona, Rima de. 1971. Polvo del camino. San José: Autores Unidos 
